\title{
GENERIC UNIQUENESS OF A MINIMAL SOLUTION FOR VARIATIONAL PROBLEMS ON A TORUS
}

\author{
ALEXANDER J. ZASLAVSKI
}

Received 17 July 2001

We study minimal solutions for one-dimensional variational problems on a torus. We show that, for a generic integrand and any rational number $\alpha$, there exists a unique (up to translations) periodic minimal solution with rotation number $\alpha$.

\section{Introduction}

In this paper, we consider functionals of the form

$$
I^{f}(a, b, x)=\int_{a}^{b} f\left(t, x(t), x^{\prime}(t)\right) d t,
$$

where $a$ and $b$ are arbitrary real numbers satisfying $a<b, x \in W^{1,1}(a, b)$ and $f$ belongs to a space of functions described below. By an appropriate choice of representatives, $W^{1,1}(a, b)$ can be identified with the set of absolutely continuous functions $x:[a, b] \rightarrow \mathbb{R}^{1}$, and henceforth we will assume that this has been done.

Denote by $\mathfrak{M}$ the set of integrands $f=f(t, x, p): \mathbb{R}^{3} \rightarrow \mathbb{R}^{1}$ which satisfy the following assumptions:

(A1) $f \in C^{3}$ and $f(t, x, p)$ has period 1 in $t, x$;

(A2) $\delta_{f} \leq f_{p p}(t, x, p) \leq \delta_{f}^{-1}$ for every $(t, x, p) \in \mathbb{R}^{3}$;

(A3) $\left|f_{x p}\right|+\left|f_{t p}\right| \leq c_{f}(1+|p|),\left|f_{x x}\right|+\left|f_{x t}\right| \leq c_{f}\left(1+p^{2}\right)$,

with some constants $\delta_{f} \in(0,1), c_{f}>0$.

Clearly, these assumptions imply that

$$
\tilde{\delta}_{f} p^{2}-\tilde{c}_{f} \leq f(t, x, p) \leq \tilde{\delta}_{f}^{-1} p^{2}+\tilde{c}_{f}
$$

for every $(t, x, p) \in \mathbb{R}^{3}$ for some constants $\tilde{c}_{f}>0$ and $0<\tilde{\delta}_{f}<\delta_{f}$.

In this paper, we analyse extremals of variational problems with integrands $f \in \mathfrak{M}$. The following optimality criterion was introduced by Aubry and Le

Copyright (C) 2002 Hindawi Publishing Corporation Abstract and Applied Analysis 7:3 (2002) 143-154 2000 Mathematics Subject Classification: 49J99, 58F99 URL: http://dx.doi.org/10.1155/S1085337502000842 
Daeron [2] in their study of the discrete Frenkel-Kontorova model related to dislocations in one-dimensional crystals.

Let $f \in \mathfrak{M}$. A function $x(\cdot) \in W_{\text {loc }}^{1,1}\left(\mathbb{R}^{1}\right)$ is called an $(f)$-minimal solution if

$$
I^{f}(a, b, y) \geq I^{f}(a, b, x)
$$

for each pair of numbers $a<b$ and each $y \in W^{1,1}(a, b)$ which satisfies $y(a)=x(a)$ and $y(b)=x(b)($ see $[2,9,10,12])$.

Our work follows Moser $[9,10]$, who studied the existence and structure of minimal solutions in the spirit of Aubry-Mather theory [2, 7].

Consider any $f \in \mathfrak{M}$. It was shown in $[9,10]$ that $(f)$-minimal solutions possess numerous remarkable properties. Thus, for every $(f)$-minimal solution $x(\cdot)$, there is a real number $\alpha$ satisfying

$$
\sup \left\{|x(t)-\alpha t|: t \in \mathbb{R}^{1}\right\}<\infty
$$

which is called the rotation number of $x(\cdot)$, and given any real $\alpha$ there exists an $(f)$-minimal solution with rotation number $\alpha$. Senn [11] established the existence of a strictly convex function $E_{f}: \mathbb{R}^{1} \rightarrow \mathbb{R}^{1}$, which is called the minimal average action of $f$ such that, for each real $\alpha$ and each $(f)$-minimal solution $x$ with rotation number $\alpha$,

$$
\left(T_{2}-T_{1}\right)^{-1} I^{f}\left(T_{1}, T_{2}, x\right) \longrightarrow E_{f}(\alpha) \text { as } T_{2}-T_{1} \longrightarrow \infty .
$$

This result is an analogue of Mather's theorem about the average energy function for Aubry-Mather sets generated by a diffeomorphism of the infinite cylinder [8].

In this paper, we show that for a generic integrand $f$ and any rational $\alpha$, there exists a unique (up to translations) $(f)$-minimal periodic solution with rotation number $\alpha$.

Let $k \geq 3$ be an integer. Set $\mathfrak{M}_{k}={\mathfrak{M} \cap C^{k}}\left(\mathbb{R}^{3}\right)$. For $f \in \mathfrak{M}_{k}$ and $q=\left(q_{1}, q_{2}, q_{3}\right) \in$ $\{0, \ldots, k\}^{3}$ satisfying $q_{1}+q_{2}+q_{3} \leq k$, we set

$$
|q|=q_{1}+q_{2}+q_{3}, \quad D^{q} f=\frac{\partial^{|q|} f}{\partial t^{q_{1}} \partial x^{q_{2}} \partial p^{q_{3}}} .
$$

For $N, \epsilon>0$ we set

$$
\begin{gathered}
E_{k}(N, \epsilon)=\left\{(f, g) \in \mathfrak{M}_{k} \times \mathfrak{M}_{k}:\left|D^{q} f(t, x, p)-D^{q} g(t, x, p)\right|\right. \\
\leq \epsilon+\epsilon \max \left\{\left|D^{q} f(t, x, p)\right|,\left|D^{q} g(t, x, p)\right|\right\} \\
\left.\forall q \in\{0,1,2\}^{3} \text { satisfying }|q| \in\{0,2\}, \forall(t, x, p) \in \mathbb{R}^{3}\right\} \\
\cap\left\{(f, g) \in \mathfrak{M}_{k} \times \mathfrak{M}_{k}:\left|D^{q} f(t, x, p)-D^{q} g(t, x, p)\right| \leq \epsilon\right. \\
\forall q \in\{0, \ldots, k\}^{3} \text { satisfying }|q| \leq k, \forall(t, x, p) \in \mathbb{R}^{3} \\
\text { such that }|p| \leq N\} .
\end{gathered}
$$


It is easy to verify that, for the set $\mathfrak{M}_{k}$ there exists a uniformity which is determined by the base $E_{k}(N, \epsilon), N, \epsilon>0$, and that the uniform space $\mathfrak{M}_{k}$ is metrizable and complete [3]. We establish the existence of a set $\mathscr{F}_{k} \subset \mathfrak{M}_{k}$ which is a countable intersection of open everywhere dense subsets of $\mathfrak{M}_{k}$ such that, for each $f \in \mathscr{F}_{k}$ and each rational $\alpha \in \mathbb{R}^{1}$, there exists a unique (up to translations) $(f)$-minimal periodic solultion with rotation number $\alpha$.

\section{Properties of minimal solutions}

Consider any $f \in \mathfrak{M}$. We note that, for each pair of integers $j$ and $k$ the translations $(t, x) \rightarrow(t+j, x+k)$ leave the variational problem invariant. Therefore, if $x(\cdot)$ is an $(f)$-minimal solution, so is $x(\cdot+j)+k$. Of course, on the torus, this represents the same curve as does $x(\cdot)$. This motivates the following terminology $[9,10]$.

We say that a function $x(\cdot) \in W_{\text {loc }}^{1,1}\left(\mathbb{R}^{1}\right)$ has no self-intersections if for all pairs of integers $j, k$ the function $t \rightarrow x(t+j)+k-x(t)$ is either always positive, or always negative, or identically zero.

Denote by $\mathbb{Z}$ the set of all integers. We have the following result (see [6, Proposition 3.2] and $[9,10])$.

Proposition 2.1. (i) Let $f \in \mathfrak{M}$. Given any real $\alpha$ there exists a nonself-intersecting $(f)$-minimal solution with rotation number $\alpha$.

(ii) For any $f \in \mathfrak{M}$ and any $(f)$-minimal solution $x$, there is the rotation number of $x$.

For each $f \in \mathfrak{M}$, each rational number $\alpha$, and each natural number $q$ satisfying $q \alpha \in \mathbb{Z}$, we define

$$
\begin{aligned}
\mathcal{N}(\alpha, q) & =\left\{x(\cdot) \in W_{\mathrm{loc}}^{1,1}\left(\mathbb{R}^{1}\right): x(t+q)=x(t)+\alpha q, t \in \mathbb{R}^{1}\right\}, \\
M_{f}(\alpha, q) & =\left\{x(\cdot) \in \mathcal{N}(\alpha, q): I^{f}(0, q, x) \leq I^{f}(0, q, y) \forall y \in \mathcal{N}(\alpha, q)\right\} .
\end{aligned}
$$

We have the following result [9, Theorems 5.1, 5.2, 5.4, and Corollaries 5.3 and 5.5].

Proposition 2.2. Let $f \in \mathfrak{M}$, let $\alpha$ be a rational number, and let $p, q \geq 1$ be integers satisfying $p \alpha, q \alpha \in \mathbb{Z}$. Then $M_{f}(\alpha, q)=M_{f}(\alpha, p) \neq \emptyset$, each $x \in M_{f}(\alpha, q)$ is a nonself-intersecting $(f)$-minimal solution with rotation number $\alpha$ and the set $M_{f}(\alpha, q)$ is totally ordered, that is, if $x, y \in M_{f}(\alpha, q)$, then either $x(t)<y(t)$ for all $t$, or $x(t)>y(t)$ for all $t$, or $x(t)=y(t)$ identically.

For any $f \in \mathfrak{M}$ and any rational number $\alpha$ we set $\mathcal{M}_{f}^{\text {per }}(\alpha)=M_{f}(\alpha, q)$, where $q$ is a natural number satisfying $q \alpha \in \mathbb{Z}$.

We have the following result (see [6, Theorem 1.1]).

Proposition 2.3. Let $f \in \mathfrak{M}$. Then there exist a strictly convex function $E_{f}: \mathbb{R}^{1} \rightarrow$ $\mathbb{R}^{1}$ satisfying $E_{f}(\alpha) \rightarrow \infty$ as $|\alpha| \rightarrow \infty$ and a monotonically increasing function $\Gamma_{f}:(0, \infty) \rightarrow[0, \infty)$ such that for each real $\alpha$, each $(f)$-minimal solution $x$ with 
rotation number $\alpha$ and each pair of real numbers $S$ and $T$,

$$
\left|I^{f}(S, S+T, x)-E_{f}(\alpha) T\right| \leq \Gamma_{f}(|\alpha|) .
$$

By Proposition 2.3 for each $f \in \mathfrak{M}$ there exists a unique number $\alpha(f)$ such that

$$
E_{f}(\alpha(f))=\min \left\{E_{f}(\beta): \beta \in \mathbb{R}^{1}\right\} .
$$

Note that assumptions (A1), (A2), and (A3) play an important role in the proofs of Propositions 2.1, 2.2, and 2.3 (see $[9,10]$ ).

\section{The main results}

TheOREM 3.1. Let $k \geq 3$ be an integer and $\alpha$ be a rational number. Then there exists a set $\mathscr{F}_{k \alpha} \subset \mathfrak{M}_{k}$ which is a countable intersection of open everywhere dense subsets of $\mathfrak{M}_{k}$ such that for each $f \in \mathfrak{M}_{k}$ the following assertions hold:

(1) If $x, y \in M_{f}^{(\text {per })}(\alpha)$, then there are integers $p$, q such that $y(t)=x(t+p)-q$ for all $t \in \mathbb{R}^{1}$.

(2) Let $x \in M_{f}^{(\text {per })}(\alpha)$ and $\epsilon>0$. Then there exists a neighborhood $U$ of $f$ in $\mathfrak{M}_{k}$ such that for each $g \in \mathcal{U}$ and each $y \in M_{g}^{(\text {per })}(\alpha)$ there are integers $p$, q such that $|y(t)-x(t+p)+q| \leq \epsilon$ for all $t \in \mathbb{R}^{1}$.

It is not difficult to see that Theorem 3.1 implies the following result.

Theorem 3.2. Let $k \geq 3$ be an integer. Then there exists a set $\mathscr{F}_{k} \subset \mathfrak{M}_{k}$ which is a countable intersection of open everywhere dense subsets of $\mathfrak{M}_{k}$ such that, for each $f \in \mathfrak{M}_{k}$ and each rational number $\alpha$ the assertions (1) and (2) of Theorem 3.1 hold.

Note that minimal solutions with irrational rotation numbers were studied in $[2,7,9,10,12]$.

\section{An auxiliary result}

Let $k \geq 3$ be an integer and $\beta \in \mathbb{R}^{1}$. For each $f \in \mathfrak{M}_{k}$, define $\mathscr{A} f \in C^{3}\left(\mathbb{R}^{3}\right)$ by

$$
(\mathscr{A f})(t, x, u)=f(t, x, u)-\beta u, \quad(t, x, u) \in \mathbb{R}^{3} .
$$

Clearly $\mathscr{A} f \in \mathfrak{M}_{k}$ for each $f \in \mathfrak{M}_{k}$.

Proposition 4.1. The mapping $\mathscr{A}: \mathfrak{M}_{k} \rightarrow \mathfrak{M}_{k}$ is continuous.

Proof. Let $f \in \mathfrak{M}_{k}$ and let $N, \epsilon>0$. In order to prove the proposition, it is sufficient to show that there exists $\epsilon_{0} \in(0, \epsilon)$ such that

$$
\mathscr{A}\left(\left\{g \in \mathfrak{M}_{k}:(f, g) \in E_{k}\left(N, \epsilon_{0}\right)\right\}\right) \subset\left\{h \in \mathfrak{M}_{k}:(h, \mathscr{A} f) \in E_{k}(N, \epsilon)\right\} .
$$

Set

$$
\Delta_{0}=2(|\beta|+1)
$$


Equation (1.2) implies that there exists $c_{0}>0$ such that

$$
\Delta_{0}|u|-c_{0} \leq f(t, x, u) \quad \forall(t, x, u) \in \mathbb{R}^{3} .
$$

Choose a number $\epsilon_{0}$ such that

$$
0<\epsilon_{0}<\min \{1, \epsilon\}, \quad 4 \epsilon_{0}+4 \epsilon_{0}\left(1-\epsilon_{0}\right)^{-1}\left(4+c_{0}\right)<\epsilon .
$$

It follows from (4.3) and (4.4) that for each $(t, x, u) \in \mathbb{R}^{3}$,

$$
\begin{aligned}
|f(t, x, u)-\beta u| & \geq|f(t, x, u)|-|\beta u| \geq|f(t, x, u)|-|\beta| \Delta_{0}^{-1}\left(f(t, x, u)+c_{0}\right) \\
& \geq|f(t, x, u)|\left(1-|\beta| \Delta_{0}^{-1}\right)-|\beta| \Delta_{0}^{-1} c_{0} \\
& \geq 2^{-1}|f(t, x, u)|-2^{-1} c_{0} .
\end{aligned}
$$

Assume that

$$
g \in \mathfrak{M}_{k}, \quad(f, g) \in E_{k}\left(N, \epsilon_{0}\right)
$$

By (1.7) and (4.7) for each $(t, x, u) \in \mathbb{R}^{3}$,

$$
\begin{gathered}
|f(t, x, u)-g(t, x, u)| \leq \epsilon_{0}+\epsilon_{0} \max \{|f(t, x, u)|,|g(t, x, u)|\}, \\
\max \{|f(t, x, u)|,|g(t, x, u)|\}-\min \{|f(t, x, u)|,|g(t, x, u)|\} \\
\leq \epsilon_{0}+\epsilon_{0} \max \{|f(t, x, u)|,|g(t, x, u)|\}, \\
\left(1-\epsilon_{0}\right) \max \{|f(t, x, u)|,|g(t, x, u)|\} \leq \min \{|f(t, x, u)|,|g(t, x, u)|\}+\epsilon_{0}, \\
|g(t, x, u)| \leq\left(1-\epsilon_{0}\right)^{-1}|f(t, x, u)|+\left(1-\epsilon_{0}\right)^{-1} \epsilon_{0} .
\end{gathered}
$$

We show that $(\mathscr{A} f, \mathscr{A} g) \in E_{k}(N, \epsilon)$. It follows from (1.7), (4.1), (4.5), and (4.7) that, for each $q=\left(q_{1}, q_{2}, q_{3}\right) \in\{0, \ldots, k\}^{3}$ satisfying $|q| \leq k$ and each $(t, x, p) \in \mathbb{R}^{3}$ satisfying $|p| \leq N$,

$$
\left|D^{q}(\mathscr{A} f)(t, x, p)-D^{q}(\mathscr{A} g)(t, x, p)\right|=\left|D^{q} f(t, x, p)-D^{q} g(t, x, p)\right| \leq \epsilon_{0}<\epsilon
$$

Let $q \in\{0,1,2\}^{3},|q| \in\{0,2\}$, and $(t, x, p) \in \mathbb{R}^{3}$. Equation (4.1) implies that

$$
\left|D^{q}(\mathscr{A} f)(t, x, p)-D^{q}(\mathscr{A} g)(t, x, p)\right|=\left|D^{q} f(t, x, p)-D^{q} g(t, x, p)\right| .
$$

If $|q|=2$, then by (1.7), (4.1), (4.5), (4.7), and (4.10),

$$
\begin{aligned}
& \left|D^{q}(\mathscr{A} f)(t, x, p)-D^{q}(\mathscr{A} g)(t, x, p)\right| \\
& \quad \leq \epsilon_{0}+\epsilon_{0} \max \left\{\left|D^{q} f(t, x, p)\right|,\left|D^{q} g(t, x, p)\right|\right\} \\
& \quad<\epsilon+\epsilon \max \left\{\left|D^{q}(\mathscr{A} f)(t, x, p)\right|,\left|D^{q}(\mathscr{A} g)(t, x, p)\right|\right\} .
\end{aligned}
$$


Assume that $q=0$. By (1.7), (4.1), (4.5), (4.6), (4.7), and (4.8),

$$
\begin{aligned}
\left|D^{q}(\mathscr{A} f)(t, x, p)-D^{q}(\mathscr{A g})(t, x, p)\right| \\
\quad=|f(t, x, p)-g(t, x, p)| \leq \epsilon_{0}+\epsilon_{0} \max \{|f(t, x, p)|,|g(t, x, p)|\} \\
\quad \leq \epsilon_{0}+\epsilon_{0} \max \left\{|f(t, x, p)|,\left(1-\epsilon_{0}\right)^{-1}|f(t, x, p)|+\left(1-\epsilon_{0}\right)^{-1} \epsilon_{0}\right\} \\
\quad=\epsilon_{0}+\epsilon_{0}\left(1-\epsilon_{0}\right)^{-1}|f(t, x, p)|+\epsilon_{0}^{2}\left(1-\epsilon_{0}\right)^{-1} \\
\quad \leq \epsilon_{0}+\epsilon_{0}^{2}\left(1-\epsilon_{0}\right)^{-1}+\epsilon_{0}\left(1-\epsilon_{0}\right)^{-1}\left[2|f(t, x, p)-\beta p|+2 c_{0}\right] \\
\quad \leq \epsilon_{0}+\epsilon_{0}^{2}\left(1-\epsilon_{0}\right)^{-1}+2 \epsilon_{0}\left(1-\epsilon_{0}\right)^{-1} c_{0}+2 \epsilon_{0}\left(1-\epsilon_{0}\right)^{-1}|f(t, x, p)-\beta p| \\
\quad \leq 2 \epsilon_{0}\left(1-\epsilon_{0}\right)^{-1}|(\mathscr{A} f)(t, x, p)|+\epsilon \leq \epsilon+\epsilon|(\mathscr{A} f)(t, x, p)| .
\end{aligned}
$$

Equations (4.9), (4.11), and (4.12) imply that $(\mathscr{A} f, \mathscr{A} g) \in E_{k}(N, \epsilon)$. Proposition 4.1 is proved.

Let $-\infty<T_{1}<T_{2}<\infty$ and $x \in W^{1,1}\left(T_{1}, T_{2}\right)$. By (4.1) we have

$$
\begin{aligned}
& I^{\mathscr{A f}}\left(T_{1}, T_{2}, x\right)=\int_{T_{1}}^{T_{2}}\left(f\left(t, x(t), x^{\prime}(t)\right)-\beta x^{\prime}(t)\right) d t \\
& =I^{f}\left(T_{1}, T_{2}, x\right)-\beta x\left(T_{2}\right)+\beta x\left(T_{1}\right) .
\end{aligned}
$$

Therefore, each $x \in W_{\text {loc }}^{1,1}\left(\mathbb{R}^{1}\right)$ is an $(\mathscr{A} f)$-minimal solution if and only if $x(\cdot)$ is an $(f)$-minimal solution.

Let $x \in W_{\text {loc }}^{1,1}\left(\mathbb{R}^{1}\right)$ be an $(f)$-minimal solution with rotation number $r$. By Proposition 2.1 there exists $c_{1}>0$ such that for all $s, t \in \mathbb{R}^{1}$,

$$
|x(t+s)-x(t)-r s| \leq c_{1} .
$$

Proposition 2.3 implies that there exists a constant $c_{2}>0$ such that for each $s \in$ $\mathbb{R}^{1}$ and each $t>0$,

$$
\begin{aligned}
\left|I^{f}(s, s+t, x)-E_{f}(r) t\right| & \leq c_{2}, \\
\left|I^{\mathscr{A} f}(s, s+t, x)-E_{\mathscr{A} f}(r) t\right| & \leq c_{2} .
\end{aligned}
$$

It follows from (4.13), (4.14), (4.15), and (4.16) that, for each $s \in \mathbb{R}^{1}$ and each $t>0$,

$$
\begin{aligned}
\left|E_{\mathscr{A} f}(r) t+\beta t r-E_{f}(r) t\right| & \\
\leq & \left|E_{\mathscr{A} f}(r) t-I^{\mathscr{A} f}(s, s+t, x)\right|+\left|I^{\mathscr{A} f}(s, s+t, x)+\beta t r-I^{f}(s, s+t, x)\right| \\
& \quad+\left|I^{f}(s, s+t, x)-E_{f}(r) t\right| \\
\leq & c_{2}+|\beta t r-\beta[x(t+s)-x(s)]|+c_{2} \leq 2 c_{2}+|\beta| c_{1} .
\end{aligned}
$$

These inequalities imply that

$$
E_{\mathscr{A} f}(r)=E_{f}(r)-\beta r \quad \forall r \in \mathbb{R}^{1} .
$$




\section{Proof of Theorem 3.1}

Let $g \in \mathfrak{M}$. We define

$$
\mu(g)=\inf \left\{\liminf _{T \rightarrow \infty} T^{-1} I^{g}(0, T, x): x(\cdot) \in W_{\text {loc }}^{1,1}([0, \infty))\right\} .
$$

In $[13$, Section 5$]$ we showed that the number $\mu(g)$ is well defined and proved the following result [13, Theorem 5.1].

Proposition 5.1. Let $f \in \mathfrak{M}$. Then there exists a constant $M_{0}>0$ such that:

(i) $I^{f}(0, T, x)-\mu(f) T \geq-M_{0}$ for each $x \in W_{\mathrm{loc}}^{1,1}([0, \infty))$ and each $T>0$.

(ii) For each $a \in \mathbb{R}^{1}$ there exists $x \in W_{\text {loc }}^{1,1}([0, \infty))$ such that $x(0)=a$ and

$$
\left|I^{f}(0, T, x)-\mu(f) T\right| \leq 4 M_{0} \quad \forall T>0 .
$$

Note that assertion (ii) of Proposition 5.1 holds by the periodicity of $f$ in $x$. Let $f \in \mathfrak{M}$. A function $x \in W_{\text {loc }}^{1,1}([0, \infty))$ is called $(f)$-good (see [5]) if

$$
\sup \left\{\left|I^{f}(0, T, x)-\mu(f) T\right|: T \in(0, \infty)\right\}<\infty .
$$

By $[6$, Theorem 4.1],

$$
E_{f}(\alpha(f))=\mu(f) \quad \forall f \in \mathfrak{M} .
$$

For $f \in \mathfrak{M}, x, y, T_{1} \in \mathbb{R}^{1}$, and $T_{2}>T_{1}$ we set

$$
U^{f}\left(T_{1}, T_{2}, x, y\right)=\inf \left\{I^{f}\left(T_{1}, T_{2}, v\right): v \in W^{1,1}\left(T_{1}, T_{2}\right), v\left(T_{1}\right)=x, v\left(T_{2}\right)=y\right\} .
$$

It is not difficult to see that for each $x, y, T_{1} \in \mathbb{R}^{1}, T_{2}>T_{1}$,

$$
\begin{gathered}
U^{f}\left(T_{1}, T_{2}, x+1, y+1\right)=U^{f}\left(T_{1}, T_{2}, x, y\right), \\
U^{f}\left(T_{1}+1, T_{2}+1, x, y\right)=U^{f}\left(T_{1}, T_{2}, x, y\right), \quad-\infty<U^{f}\left(T_{1}, T_{2}, x, y\right)<\infty, \\
\inf \left\{U^{f}\left(T_{1}, T_{2}, a, b\right): a, b \in \mathbb{R}^{1}\right\}>-\infty .
\end{gathered}
$$

Denote by $\mathfrak{M}_{\text {per }}$ the set of all $f \in \mathfrak{M}$ such that $\alpha(f)$ is rational and denote by $\mathfrak{M}_{\text {per }}^{0}$ the set of all $g \in \mathfrak{M}_{\text {per }}$ for which there exist an $(g)$-minimal solution $w \in C^{2}\left(\mathbb{R}^{1}\right)$, a continuous function $\pi: \mathbb{R}^{1} \rightarrow \mathbb{R}^{1}$, and integers $m, n$ such that the following properties hold:

(P1) $\pi(x+1)=\pi(x), x \in \mathbb{R}^{1}$;

(P2) $n \geq 1$ and $\alpha(g)=m n^{-1}$ is an irreducible fraction;

(P3) $w(t+n)=w(t)+m$ for all $t \in \mathbb{R}^{1}$;

(P4) $U^{g}(0,1, x, y)-\mu(g)-\pi(x)+\pi(y) \geq 0$ for each $x, y \in \mathbb{R}^{1}$;

(P5) for any $u \in W^{1,1}(0, n)$, the equality

$$
I^{g}(0, n, u)=n \mu(g)+\pi(u(0))-\pi(u(n))
$$

holds if and only if there are integers $i, j$ such that $u(t)=w(t+i)-j$ for all $t \in[0, n]$. 
Consider the manifold $\left(\mathbb{R}^{1} / \mathbb{Z}\right)^{2}$ and the canonical mapping $P: \mathbb{R}^{2} \rightarrow\left(\mathbb{R}^{1} / \mathbb{Z}\right)^{2}$. We have the following result [13, Proposition 6.2].

Proposition 5.2. Let $\Omega$ be a closed subset of $\left(\mathbb{R}^{1} / \mathbb{Z}\right)^{2}$. Then there exists a bounded nonnegative function $\phi \in C^{\infty}\left(\left(\mathbb{R}^{1} / \mathbb{Z}\right)^{2}\right)$ such that

$$
\Omega=\left\{x \in\left(\mathbb{R}^{1} / \mathbb{Z}\right)^{2}: \phi(x)=0\right\} .
$$

Proposition 5.2 is proved by using [1, Chapter 2, Section 3, Theorem 1] and the partition of unity (see [4, Appendix 1]).

We also have the following result (see [13, Proposition 6.3]).

Proposition 5.3. Suppose that $f \in \mathfrak{M}_{\text {per }}, \alpha(f)=m n^{-1}$ is an irreducible fraction ( $m, n$ are integers, $n \geq 1$ ) and $w \in W_{\text {loc }}^{1,1}\left(\mathbb{R}^{1}\right)$ is an $(f)$-minimal solution satisfying $w(t+n)=w(t)+m$ for all $t \in \mathbb{R}^{1}$. Let $\phi \in C^{\infty}\left(\left(\mathbb{R}^{1} / \mathbb{Z}\right)^{2}\right)$ be as guaranteed in Proposition 5.2 with

$$
\Omega=\{P(t, w(t)): t \in[0, n]\},
$$

and let

$$
g(t, x, p)=f(t, x, p)+\phi(P(t, x)), \quad(t, x, p) \in \mathbb{R}^{3} .
$$

Then $g \in \mathfrak{M}_{\text {per }}^{0}$ and there is a continuous function $\pi: \mathbb{R}^{1} \rightarrow \mathbb{R}^{1}$ such that the properties (P1), (P2), (P3), (P4), and (P5) hold with $g, w, \pi, m, n$ and $\alpha(g)=\alpha(f)$.

In the sequel we need the following two lemmas proved in [13].

Lemma 5.4 [13, Lemma 6.6]. Assume that $k \geq 3$ is an integer, $g \in \mathfrak{M}_{\text {per }}^{0} \cap \mathfrak{M}_{k}$, and properties (P1), (P2), (P3), (P4), and (P5) hold with a g-minimal solution $w(\cdot) \in C^{2}\left(\mathbb{R}^{1}\right)$, a continuous function $\pi: \mathbb{R}^{1} \rightarrow \mathbb{R}^{1}$ and integers $m, n$. Then for each $\epsilon \in(0,1)$, there exists a neighborhood $\mathcal{U}$ of $g$ in $\mathfrak{M}_{k}$ such that for each $h \in \mathcal{U}$ and each $(h)$-good function $v \in W_{\text {loc }}^{1,1}([0, \infty))$ there are integers $p$, q such that

$$
|v(t)-w(t+p)-q| \leq \epsilon \quad \text { for all large enough } t
$$

Lemma 5.5 [13, Corollary 6.1]. Assume that $k \geq 3$ is an integer, $g \in \mathfrak{M}_{\text {per }}^{0} \cap \mathfrak{M}_{k}$, and properties (P1), (P2), (P3), (P4), and (P5) hold with a g-minimal solution $w(\cdot) \in C^{2}\left(\mathbb{R}^{1}\right)$, a continuous function $\pi: \mathbb{R}^{1} \rightarrow \mathbb{R}^{1}$ and integers $m, n$. Then there exist a neighborhood $U$ of $g$ in $\mathfrak{M}_{k}$ and a number $L>0$ such that for each $h \in \mathcal{U}$ and each $(h)$-good function $v \in W_{\text {loc }}^{1,1}([0, \infty))$, the following property holds.

There is a number $T_{0}>0$ such that

$$
\left|v\left(t_{2}\right)-v\left(t_{1}\right)-\alpha(g)\left(t_{2}-t_{1}\right)\right| \leq L
$$

for each $t_{1} \geq T_{0}$ and each $t_{2}>t_{1}$. 
Completion of the proof of Theorem 3.1. Let $k \geq 3$ be an integer and let $\alpha=$ $m n^{-1}$ be an irreducible fraction ( $n \geq 1$ and $m$ are integers). Let $f \in \mathfrak{M}_{k}$. By Proposition 2.2 there exists an $(f)$-minimal solution $w_{f}(\cdot) \in W_{\text {loc }}^{1,1}\left(\mathbb{R}^{1}\right)$ such that

$$
w_{f}(t+n)=w_{f}(t)+m \quad \forall t \in \mathbb{R}^{1} .
$$

Choose

$$
\beta \in \partial E_{f}(\alpha)
$$

Consider a mapping $\mathscr{A}: \mathfrak{M}_{k} \rightarrow \mathfrak{M}_{k}$ defined by (4.1). By Proposition 4.1 the mapping $\mathscr{A}$ is continuous. Clearly there exists a continuous $\mathscr{A}^{-1}: \mathfrak{M}_{k} \rightarrow \mathfrak{M}_{k}$. Equations (5.14) and (4.18) imply that

$$
0 \in \partial E_{\mathscr{A} f}(\alpha), \quad E_{\mathscr{A} f}(\alpha)=\min \left\{E_{\mathscr{A} f}(r): r \in \mathbb{R}^{1}\right\}=\mu(\mathscr{A} f)
$$

and that $\mathscr{A} f \in \mathfrak{M}_{\text {per }}$. It follows from Proposition 5.2 that there exists a bounded nonnegative function $\phi \in C^{\infty}\left(\left(\mathbb{R}^{1} / \mathbb{Z}\right)^{2}\right)$ such that

$$
\left\{x \in\left(\mathbb{R}^{1} / \mathbb{Z}\right)^{2}: \phi(x)=0\right\}=\left\{P\left(t, w_{f}(t)\right): t \in[0, n]\right\} .
$$

Set $f^{(\beta)}=\mathscr{A} f$ and for each $\gamma \in(0,1)$ define

$$
f_{\gamma}(t, x, u)=f(t, x, u)+\gamma \phi(P(t, x)), \quad(t, x, u) \in \mathbb{R}^{3}, \quad f_{\gamma}^{(\beta)}=\mathscr{A}\left(f_{\gamma}\right) .
$$

Proposition 5.3 implies that for each $\gamma \in(0,1)$,

$$
\begin{gathered}
f_{\gamma}^{(\beta)} \in \mathfrak{M}_{\text {per }}^{0} \cap \mathfrak{M}_{k}, \\
f_{\gamma} \longrightarrow f \quad \text { as } \gamma \longrightarrow 0^{+}, \quad f_{\gamma}^{(\beta)} \longrightarrow f^{\beta)} \quad \text { as } \gamma \longrightarrow 0^{+} \text {in } \mathfrak{M}_{k} .
\end{gathered}
$$

Fix $\gamma \in(0,1)$ and an integer $n \geq 1$. By Proposition 5.3 the properties (P1), (P2), (P3), (P4), and (P5) hold with $g=f_{\gamma}^{(\beta)}, \alpha(g)=\alpha$ and $w(\cdot)=w_{f}$.

By Lemmas 5.4 and 5.5, there exists an open neighborhood $V(f, \gamma, n)$ of $f_{\gamma}^{(\beta)}$ in $\mathfrak{M}_{\gamma}$ and a number $L(f, \gamma, n)>0$ such that the following properties hold:

(i) for each $h \in V(f, \gamma, n)$ and each $(h)$-good function $v \in W_{\text {loc }}^{1,1}([0, \infty))$, there are integers $p, q$ such that

$$
\left|v(t)-w_{f}(t+p)-q\right| \leq \frac{1}{n}
$$

for all large enough $t$;

(ii) for each $h \in V(f, \gamma, n)$ and each $(h)$-good function $v \in W_{\text {loc }}^{1,1}([0, \infty))$, there is a number $T_{0}$ such that

$$
\left|v\left(t_{2}\right)-v\left(t_{1}\right)-\alpha\left(f_{\gamma}^{(\beta)}\right)\left(t_{2}-t_{1}\right)\right| \leq L
$$

for each $t_{1} \geq T_{0}$ and each $t_{2}>t_{1}$. 
Let $h \in V(f, \gamma, n)$ and let $v \in W_{\text {loc }}^{1,1}\left(\mathbb{R}^{1}\right)$ be an $(h)$-minimal solution with rotation number $\alpha(h)$. Then by Proposition 2.3, (2.3), (5.4), and property (ii), $\left.v\right|_{[0, \infty)}$ is an $(h)$-good function and there is $T_{0}$ such that (5.20) holds for each $t_{1} \geq T_{0}$ and each $t_{2}>t_{1}$. Since $v \in W_{\text {loc }}^{1,1}\left(\mathbb{R}^{1}\right)$ has rotation number $\alpha(h)$ it follows from Proposition 2.1 that there exists $c_{1}>0$ such that

$$
|v(t+s)-v(t)-\alpha(h) s| \leq c_{1} \quad \forall s, t \in \mathbb{R}
$$

Equations (5.15), (5.17), (5.20), and (5.21) imply that

$$
\alpha(h)=\alpha\left(f_{\gamma}^{(\beta)}\right)=\alpha\left(f^{(\beta)}\right)=\alpha .
$$

Thus we have shown that

$$
\alpha(h)=\alpha \quad \forall h \in V(f, \gamma, n)
$$

Let $h \in V(f, \gamma, n)$ and let $v \in W_{\text {loc }}^{1,1}\left(\mathbb{R}^{1}\right)$ be an $(h)$-minimal solution with rotation number $\alpha$. It follows from Proposition 2.3, (2.3), and (5.4) that $\left.v\right|_{[0, \infty)}$ is an $(h)$-good function. By property (i) there exist integers $p, q$ such that

$$
\left|v(t)-w_{f}(t+p)-q\right| \leq \frac{1}{n} \quad \text { for all large enough } t .
$$

Therefore we proved the following property:

(iii) for each $h \in V(f, \gamma, n)$ and each $(h)$-minimal solution $v \in \mathcal{M}_{h}^{\text {per }}(\alpha)$, there exist integers $p, q$ such that

$$
\left|v(t)-w_{f}(t+p)-q\right| \leq \frac{1}{n} \quad \forall t \in \mathbb{R}^{1} .
$$

Define

$$
\mathcal{U}(f, \gamma, n)=\mathscr{A}^{-1}(V(f, \gamma, n))
$$

Clearly $\mathcal{U}(f, \gamma, n)$ is an open neighborhood of $f_{\gamma}$ in $\mathfrak{M}_{k}$. By property (iii) the following property holds:

(iv) for each $\xi \in \mathcal{U}(f, \gamma, n)$ and each $(\xi)$-minimal solution $v \in \mathcal{M}_{\xi}^{\text {per }}(\alpha)$, there exist integers $p, q$ such that (5.25) holds.

Define

$$
\mathscr{F}_{k \alpha}=\cap_{n=1}^{\infty} \cup\left\{\mathcal{U}(f, \gamma, i): f \in \mathfrak{M}_{k}, \gamma \in(0,1), i \geq n\right\} .
$$

It is not difficult to see that $\mathscr{F}_{k \alpha}$ is a countable intersection of open everywhere dense subsets of $\mathfrak{M}_{k}$. 
Let $g \in \mathscr{F}_{k \alpha}, \epsilon \in(0,1)$ and $x, y \in \mathcal{M}_{g}^{(\mathrm{per})}(\alpha)$. Choose a natural number $n>8 \epsilon^{-1}$. By (5.27) there exist $f \in \mathfrak{M}_{k}, \gamma \in(0,1)$ and an integer $i \geq n$ such that

$$
g \in U(f, \gamma, i)
$$

It follows from (5.28) and property (iv) that there exist integers $p_{1}, q_{1}, p_{2}, q_{2}$ such that

$$
\begin{array}{ll}
\left|x(t)-w_{f}\left(t+p_{1}\right)-q_{1}\right| \leq \frac{1}{i} & \forall t \in \mathbb{R}^{1}, \\
\left|y(t)-w_{f}\left(t+p_{2}\right)-q_{2}\right| \leq \frac{1}{i} & \forall t \in \mathbb{R}^{1},
\end{array}
$$

where $w_{f} \in M_{f}^{(\mathrm{per})}(\alpha)$.

It follows from (5.29) and (5.30) that for all $t \in \mathbb{R}^{1}$,

$$
\begin{aligned}
\left|x\left(t-p_{1}\right)-w_{f}(t)-q_{1}\right| & \leq \frac{1}{i}, \\
\left|y\left(t-p_{2}\right)-w_{f}(t)-q_{2}\right| & \leq \frac{1}{i}, \\
\left|x\left(t-p_{1}-q_{1}\right)-\left(y\left(t-p_{2}\right)-q_{2}\right)\right| & \leq \frac{2}{i}, \\
\left|x\left(t+p_{2}-p_{1}\right)-y(t)-q_{1}+q_{2}\right| & \leq \frac{2}{i} \leq \frac{2}{n}<\epsilon .
\end{aligned}
$$

Since $\epsilon$ is any number in $(0,1)$, we conclude that there exist integers $p, q$ such that

$$
x(t+p)-q=y(t) \quad \forall t \in \mathbb{R}^{1} .
$$

Assume that $h \in \mathcal{U}(f, \gamma, i)$ and $z \in M_{h}^{(\text {per })}(\alpha)$. By the property (iv) there exist integers $p_{3}, q_{3}$ such that

$$
\left|z(t)-w_{f}\left(t+p_{3}\right)-q_{3}\right| \leq \frac{1}{i} \quad \forall t \in \mathbb{R}^{1} .
$$

Combined with (5.29) this inequality implies that

$$
\left|z\left(t-p_{3}\right)-q_{3}-x\left(t-p_{1}\right)+q_{1}\right| \leq \frac{2}{i} \leq \frac{2}{n}<\epsilon
$$

for all $t \in \mathbb{R}^{1}$. This completes the proof of Theorem 3.1.

\section{References}

[1] J.-P. Aubin and I. Ekeland, Applied Nonlinear Analysis, John Wiley \& Sons, New York, 1984.

[2] S. Aubry and P. Y. Le Daeron, The discrete Frenkel-Kontorova model and its extensions. I. Exact results for the ground-states, Phys. D 8 (1983), no. 3, 381-422. 
[3] J. L. Kelley, General Topology, Van Nostrand, New York, 1955.

[4] S. Kobayashi and K. Nomizu, Foundations of Differential Geometry. Vol. I, Interscience Publishers, New York, 1963.

[5] A. Leizarowitz, Infinite horizon autonomous systems with unbounded cost, Appl. Math. Optim. 13 (1985), no. 1, 19-43.

[6] A. Leizarowitz and A. J. Zaslavski, Infinite-horizon variational problems with nonconvex integrands, SIAM J. Control Optim. 34 (1996), no. 4, 1099-1134.

[7] J. N. Mather, Existence of quasiperiodic orbits for twist homeomorphisms of the annulus, Topology 21 (1982), no. 4, 457-467.

[8] , Minimal measures, Comment. Math. Helv. 64 (1989), no. 3, 375-394.

[9] J. Moser, Minimal solutions of variational problems on a torus, Ann. Inst. H. Poincaré Anal. Non Linéaire 3 (1986), no. 3, 229-272.

[10] - Recent developments in the theory of Hamiltonian systems, SIAM Rev. 28 (1986), no. 4, 459-485.

[11] W. Senn, Strikte Konvexität für Variationsprobleme auf dem n-dimensionalen torus, Manuscripta Math. 71 (1991), no. 1, 45-65 (German).

[12] A. J. Zaslavski, Ground states in Frenkel-Kontorova model, Math. USSR-Izv. 29 (1987), 323-354.

[13] _ Existence and structure of extremals for one-dimensional nonautonomous variational problems, J. Optim. Theory Appl. 97 (1998), no. 3, 731-757.

Alexander J. Zaslavski: Department of Mathematics, Technion-Israel Institute of Technology, Haifa 32000, IsRael

E-mail address: ajzasl@techunix.technion.ac.il 


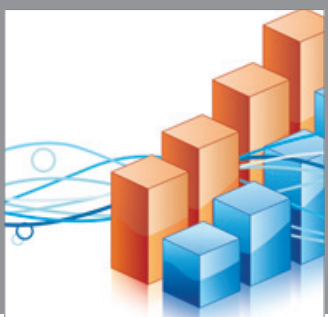

Advances in

Operations Research

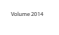

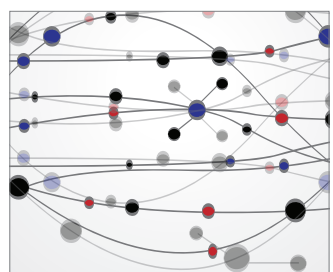

\section{The Scientific} World Journal
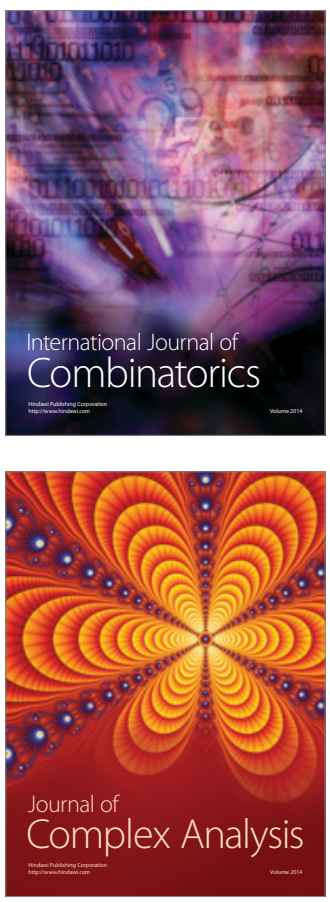

International Journal of

Mathematics and

Mathematical

Sciences
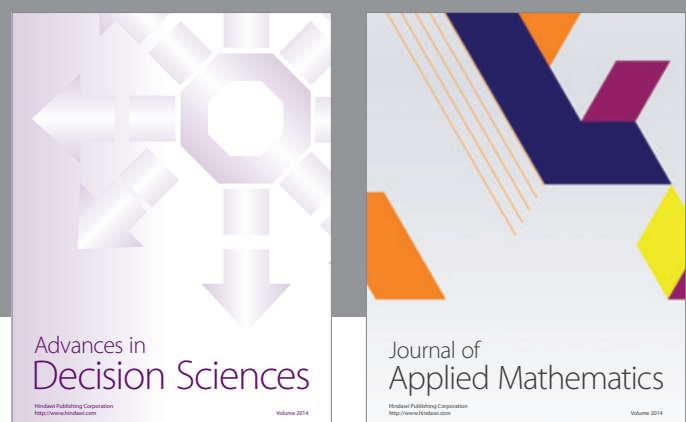

Journal of

Applied Mathematics
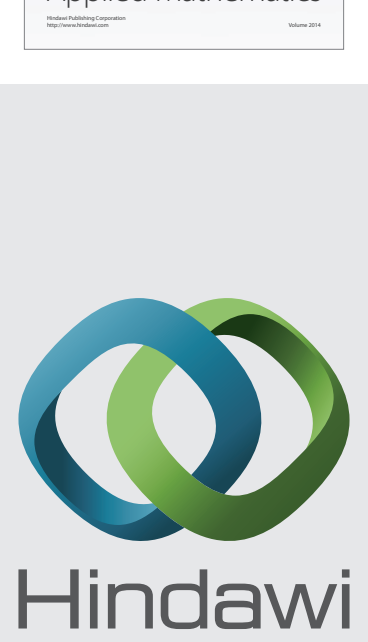

Submit your manuscripts at http://www.hindawi.com
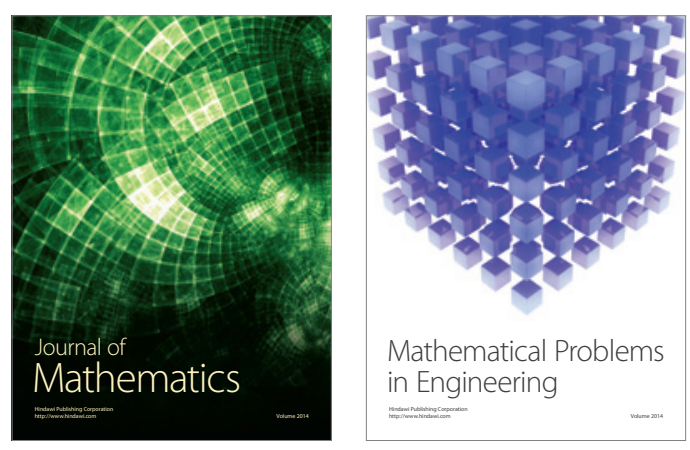

Mathematical Problems in Engineering
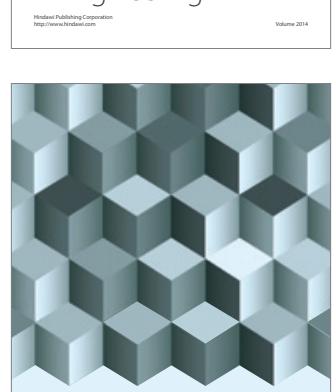

Journal of

Function Spaces
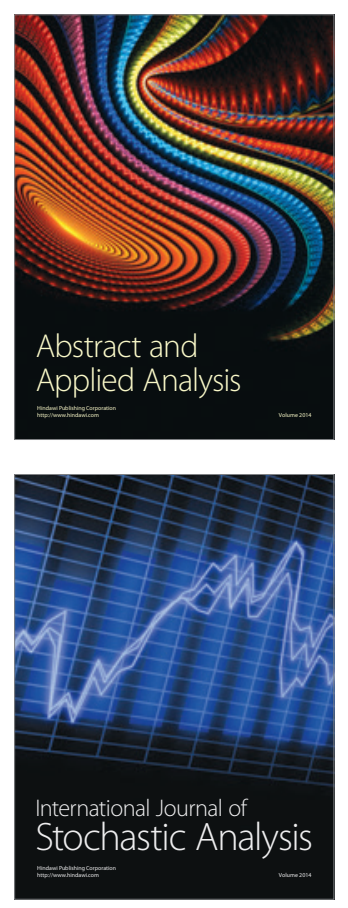

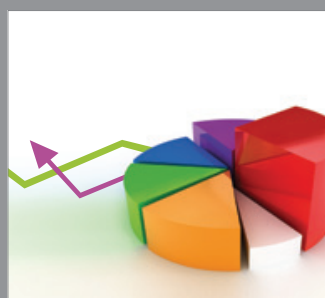

ournal of

Probability and Statistics

Promensencen
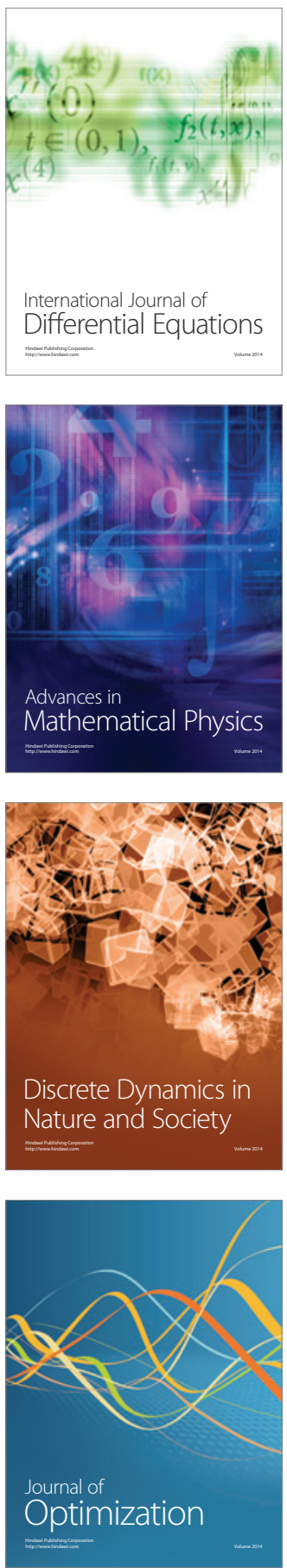